\title{
Mechanical Properties in Kinematics Based on Pulse Coupled Neural Network
}

\author{
Yali $\mathrm{Xu}^{1}$, Kaihua $\mathrm{Xu} \mathrm{u}^{2}$ \\ ${ }^{1}$ School of Physical Education, Jiangxi University of Traditional Chinese Medicine, Nanchang, \\ 330004, China \\ ${ }^{2}$ Police Training Management Department, Jiangxi People's Police College, Nanchang, 330103, \\ China \\ yali_xu2013@yeah.net
}

Keywords: Aerobics, Movement analysis, Biological vision; Multi core DSP, Low power consumption, Bandwidth.

\begin{abstract}
In order to improve the efficiency of aerobics movement image analysis, on the basis of biological vision and image processing technology, this paper designs the image motion analysis system with the core of DSP+FPGA circuit and the high-speed external memory allocation. This system is based on the image segmentation and clustering algorithm as the theoretical model to ensure the system having enough computing power on the hardware, at the same time providing sufficient bandwidth for the large amount of image transmission. The test shows that the image processing system has reached the real-time and stability of aerobics movement analysis requirement, further realizing low power consumption and improving the efficiency of image processing algorithm.
\end{abstract}

\section{Introduction}

High performance digital signal processor is a special digital signal processor, which is very suitable for embedded image processing. The aerobics movement elements analysis needs to combine with the continuous of images to present the beauty of art, so it has high requirement on the real-time and efficiency of image processing [1,2]. This paper presents a real time image processing system with three high performance DSP core circuit. On the basis of the a plurality of DSP parallel running, it meets the real time image processing and further realizes low power consumption. The upgrade performance provides a broader space for the image processing algorithm, and the overall frame is as shown in Figure 1.

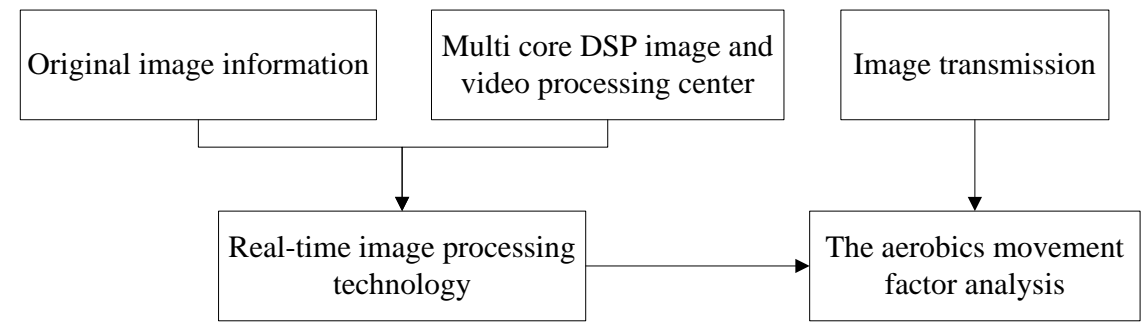

Fig.1: The multi-core processor design for aerobics movement factor analysis

Figure 1 shows the multi-core processor framework of aerobics movement decomposition and element analysis. Its core part is DSP, using the Harvard architecture or modified Harvard architecture. It separates storage space of data and program code, uses the independent address and data bus. The improved Harvard architecture allows data exchange between code storage space and data storage space, so the system has fast calculation speed, high precision and high capability. 


\section{DSP Multi-core Optimization Processing Algorithm Design for Action Element Analysis}

Aerobics movements can reflect the beauty of aerobics, which is the key of aerobics art design. As shown in Figure 2 it is a classic aerobics art image [3, 4]. From the image it can be seen, the dance movements are mainly embodied in five aspects.

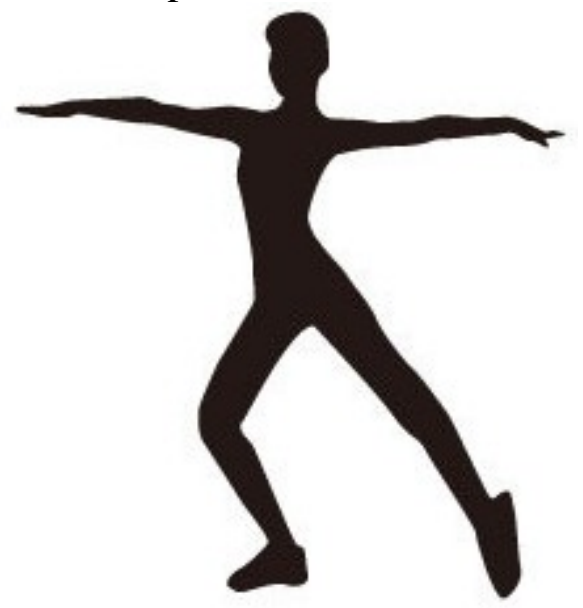

Fig.2: The diagram of aerobics movement

Through the action element analysis technique, the image is segmented, and each frame of image can be clustered. The clustering method used in this paper is the Kmeans clustering algorithm, and the clustering center can be expressed as:

$$
D=\frac{\sum_{j=1}^{m} \sum_{a_{i} \in b_{i}} K\left(a_{i}, b_{i}\right)}{\sum_{j=1}^{m}\left|B_{i}\right|} .
$$

$K$ is distance function; $m$ indicates the number of computing image cluster. And the similarity between the two frames is computed as follows:

$$
S(u, v)=\left\{\begin{array}{c}
g(u, v), \text { if } g(u, v)>2 \\
0
\end{array} .\right.
$$

Each frame of $g$ can be expressed as:

$$
g(u, v)=\sum_{p=1}^{5} g_{p}(u, v)
$$

Then the similarity relation can be expressed as:

$$
g_{p}(u, v)=\left\{\begin{array}{ccc}
1 & \text { if } & u_{p}=g_{p} \\
& 0
\end{array} .\right.
$$

$u_{p}, g_{p}$ are corresponding values of $P$ item in $u, g ; g(u, v)$ is the five postures similarity of aerobics technical action recognition. After merging the frame, the aerobics movement factors decomposition model can be obtained, as shown in the formula (5)

$$
H^{p}(t)=\bigcup_{r=1}^{K} T_{r}^{p}(t), p=1,2, \cdots, 5 \quad 1 \leq t \leq l .
$$

$T$ is sample length, $l$ is difference length. In order to realize the algorithm, this paper adopts a multi-core DSP computer and uses the MATLAB software to program the algorithm [5]. The main program of picture import process is as follows:

[filename,pathname]=uigetfile(\{'*.jpg';'*.bmp';'*tif';'***'\},'openfile');

if isequal(filename,0)|isequal(pathname,0)

errordlg('no file','error');

return; 


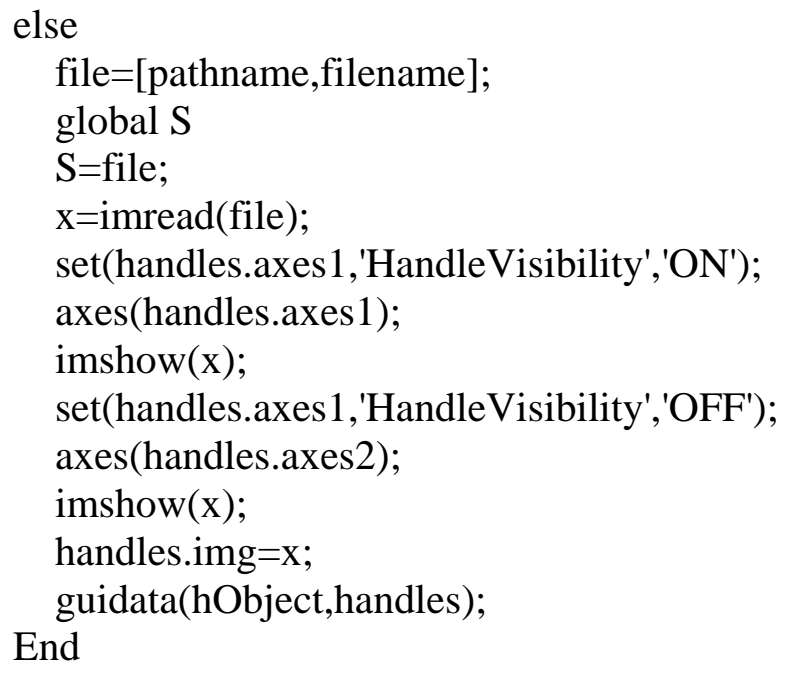

\section{DSP Processing Algorithm of Aerobics Action Elements Analysis based on Multi-core}

In order to verify the effectiveness and reliability of image action elements segmentation and real time process algorithm designed in second section, this paper uses the multi-core DSP processing computer to segment aerobics image [6-8]. The fundamental connection form of multinuclear DSP is as shown in Figure 3.

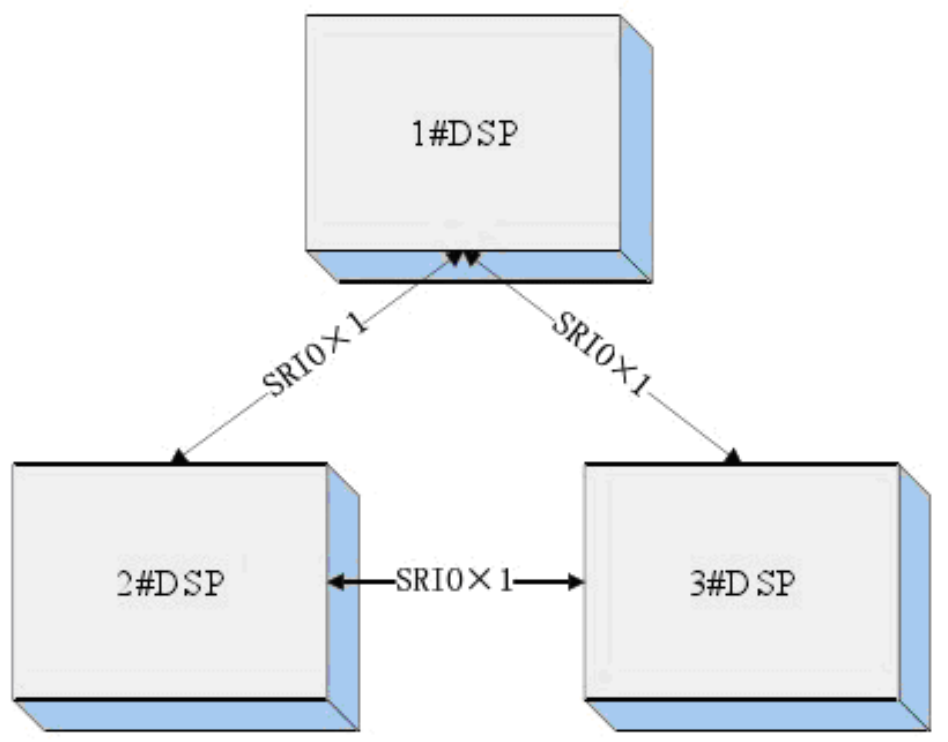

Fig.3: Multi core DSP interconnections

Figure 3 shows three DSP interconnection links form. It mainly uses the SRIO*1 to interconnect, and uses the model to connect with each other, so as to form a flexible parallel structure, which provides a very flexible space for the parallel task allocation.

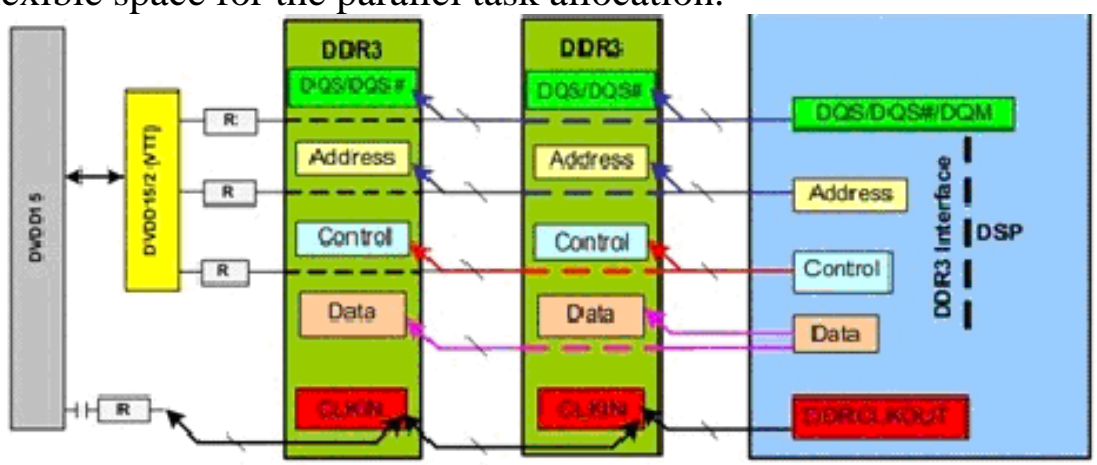

Fig.4: The DDR3 topology 
As shown in Figure 4 it indicates the storage topology structural form. The core DSP of real time image processing system has high speed chip memory, so that data transmission rate is up to $1333 \mathrm{MHz}$ [9]. The system configures two 16bit memories, so that the data width is broadened to 32bit.

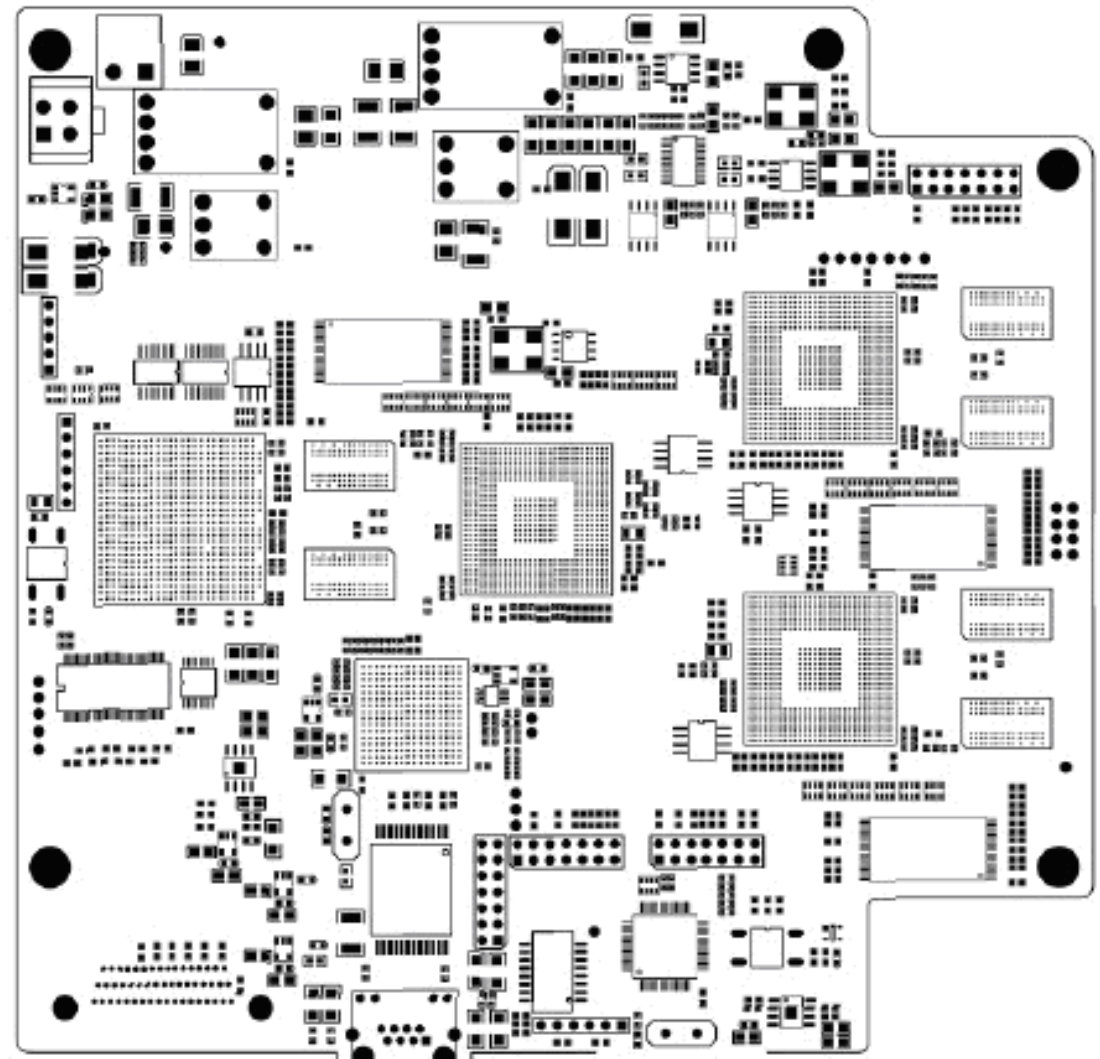

Fig.5: The system circuit board

The DSP processing board in multi-core DSP system is as shown in Figure 5 [10]. The processing board size in multi-core DSP system is reduced nearly half than before, saving the manufacturing cost of printed circuit board and greatly improving the calculation processing speed.

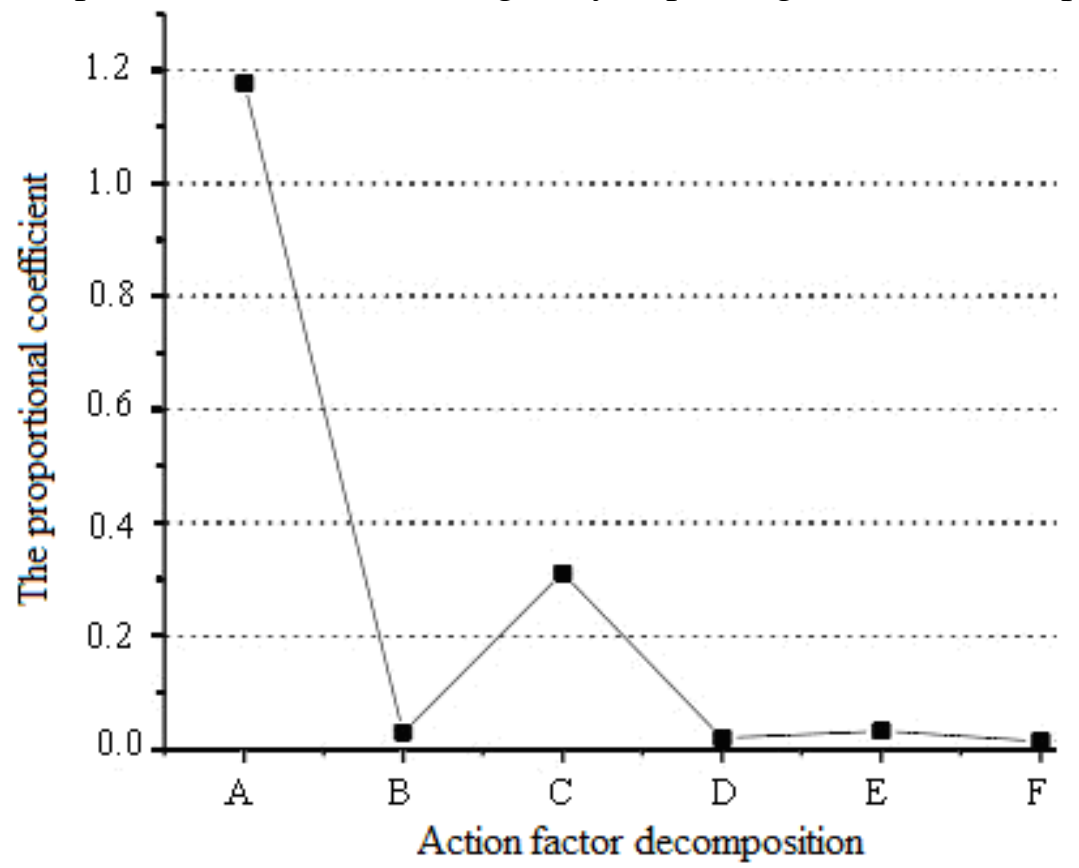

Fig.6: Action element decomposition ratio coefficient

As shown in Figure 6, the order of affecting the aesthetic action elements is $A \rightarrow B \rightarrow C \rightarrow D \rightarrow E \rightarrow F$, namely the $A$ action changes obviously, which is the main affect factors of the aerobics beauty [11]. There is no so high proportion of elements in continuous actions. 


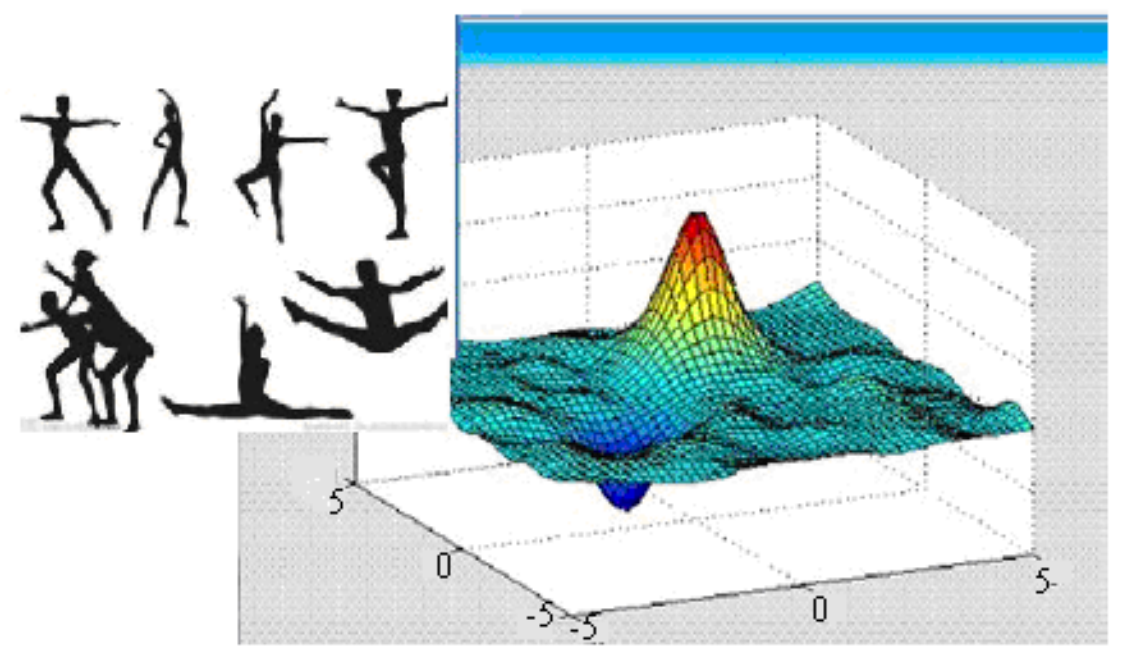

Fig.7: The aerobics movement factor analysis

Figure 7 shows the aerobics movement element analysis results [12]. In the continuous action, there is the best part of aesthetic embodiment, this part uses the 3D images to show, as shown the spike part in Figure 7, the part indicates the embodied elements of aerobics beauty, which can be stressed designed.

\section{Summary}

According to the biological visual aesthetic sensibility, combined with aerobics art form, the aerobics image is divided into five main parts, so as to realize the aerobics art image segmentation, which prepares the conditions for the action element analysis. In order to improve the efficiency of image analysis and achieve the image effective segmentation and fusion using clustering algorithm, this paper designs the multi-core DSP image analysis system, and uses the DDR3 topology structure to broaden the data width, which improves the efficiency of data transmission. Through the system testing, the image processing can perfectly complete aerobics movement factors analysis, and can get the proportion of motion analysis continuity, which can reduce board size, and power consumption.

\section{Acknowledgments}

The work was supported by the teaching reform research project in Jiangxi University of traditional Chinese Medicine with the project number 2013jzyb-30 and the project name Experimental Study on Cooperative Inquiry Teaching Method in Technical Teaching of Traditional Chinese Medicine Health Qigong classes.

\section{References}

[1] B.J. Zou, H.Y. Zhou, L. Wang, Y.X. Liang. Fusion and splicing of 3D grid in the overlap area. Acta electronic Sinica, 2012, 4(5): 13-16.

[2] Y.C. Yang, J. W. Dang, Y.P. Wang. Medical image fusion method based on adaptive PCNN and lifting wavelet transform. Journal of Computer Aided Design \& computer graphics, 2012 4(4): 42-45.

[3] Q. Liu, X.S. Ma, L.J. Zhang, Y.D. Ma, Z. Dong. Color image automatic segmentation algorithm based on vector PCNN. Computer applications and software, 203, 2(12): 61-64.

[4] J.F. Li, B.J. Zou, G.J. Xin, L.Z. Li M. L. Cai. An adaptive PCNN image fusion method. Journal of Central South University (NATURAL SCIENCE EDITION), 2012, 5(7): 23-26.

[5] H.B. Lin, B. Liu, Y.C. Zhang. Scattered point cloud variable scale algorithm in the reverse engineering. Journal of research on mechanical engineering, 2013, 3(14): 53-56. 
[6] G.J. Xin, B.J. Zou, J.F. Li, Z.L. Chen, M.L. Cai. The image segmentation combined with the maximum variance ratio and PCNN model. China image graphics, 2012, 3(7): 75-78.

[7] H.T. Zhang. Development trend and application of digital signal processor. Silicon Valley, 2013, 4(23): 6-9.

[8] Z.C. Zhou, Z.N. Shen. The design method of power supply in high speed embedded system. Microcontroller and embedded system application, 2012, 4(3): 101-104.

[9] C. Cheng, X.Y. Cheng, D.Y. Bi, W. Zhu. High speed DSP system signal integrity simulation based on HyperLynx. Electronic devices, 2012, 3(2): 62-64.

[10] Z.H. Zhao, H. Zheng. The embedded reconfigurable multi DSP image parallel processing system. Microcontroller and embedded system application, 2013, 4(2): 72-74.

[11] Wang Jun, Zhang Yuxi, Li Wei. High performance DSP connection method. Electronic devices, 2012, 2(6): 112-114.

[12] Y. Zheng, X.D. Tao. Multi DSP parallel computing structure of high speed real time signal processing system. Technology information, 2012, 4(34): 36-39. 